\title{
A PROGRAMAÇÃO MATEMÁTICA, A TECNOLOGIA E A MAIS-VALIA
}

\author{
Sdepan Bogosian Neto \\ Centro de Projetos de Navios - Marinha do Brasil \\ Ilha das Cobras, Ed. 16, s/no. Centro, Rio de Janeiro CEP 20091-000 \\ e-mail: sbogosian@gmail.com
}

\begin{abstract}
RESUMO
Este artigo utiliza a Programação Matemática para analisar a influência da Mais-Valia e da Tecnologia na composição do lucro. Para este fim, faz uso da Teoria do Valor-Trabalho, Fronteiras de Possibilidades de Produção e multiplicadores de Lagrange.

Palavras-chave: Programação Matemática, Lucro, Mais-Valia, Fronteiras de Possibilidade de Produção.
\end{abstract}

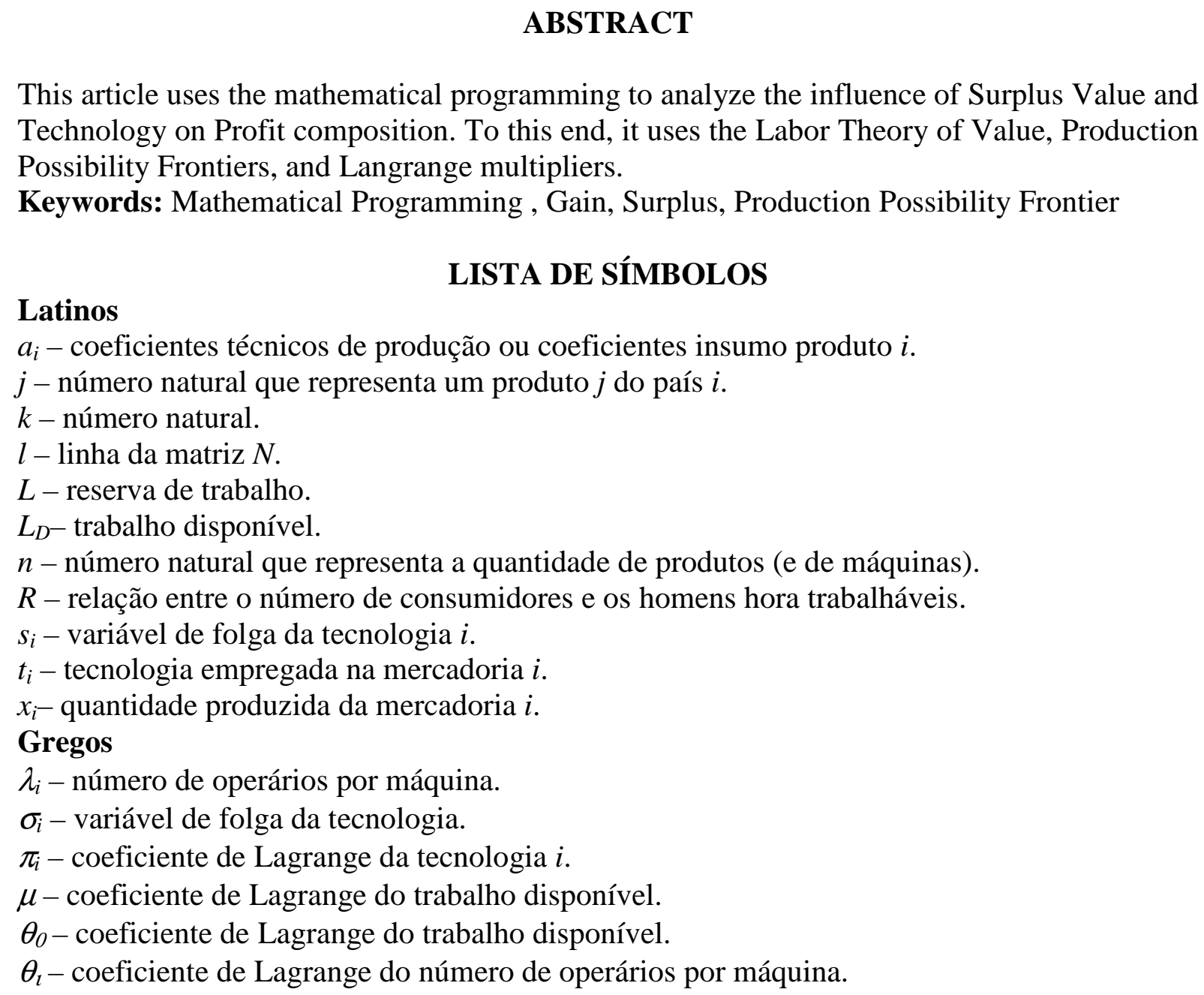

ABSTRACT

This article uses the mathematical programming to analyze the influence of Surplus Value and Technology on Profit composition. To this end, it uses the Labor Theory of Value, Production Possibility Frontiers, and Langrange multipliers.

Keywords: Mathematical Programming, Gain, Surplus, Production Possibility Frontier

\section{Latinos}

\section{LISTA DE SÍMBOLOS}

$a_{i}-$ coeficientes técnicos de produção ou coeficientes insumo produto $i$.

$j$ - número natural que representa um produto $j$ do país $i$.

$k$ - número natural.

$l$ - linha da matriz $N$.

$L$ - reserva de trabalho.

$L_{D^{-}}$trabalho disponível.

$n$ - número natural que representa a quantidade de produtos (e de máquinas).

$R$ - relação entre o número de consumidores e os homens hora trabalháveis.

$s_{i}$ - variável de folga da tecnologia $i$.

$t_{i}$ - tecnologia empregada na mercadoria $i$.

$x_{i}$ - quantidade produzida da mercadoria $i$.

Gregos

$\lambda_{i}$ - número de operários por máquina.

$\sigma_{i}$ - variável de folga da tecnologia.

$\pi_{i}$ - coeficiente de Lagrange da tecnologia $i$.

$\mu$ - coeficiente de Lagrange do trabalho disponível.

$\theta_{0}$ - coeficiente de Lagrange do trabalho disponível.

$\theta_{l}$ - coeficiente de Lagrange do número de operários por máquina.

\section{LISTA DE ABREVIATURAS E SIGLAS}

FPP - fronteira de possibilidade de produção.

PPL - programa de programação linear. 


\section{INTRODUÇÃO}

O lucro na economia Ricardiana se explicava pela diferença entre o pagamento dos salários e da terra e o preço de venda dos produtos (WIKIPEDIA, 2013). A este termo, mais-valia, ou "surplus" já recebia a atenção de Adam Smith (ibid.):

Ao analisar a gênese do lucro capitalista, Marx toma como ponto de partida as categorias da Escola Clássica Inglesa: já Adam Smith havia observado que o trabalho incorporado em uma mercadoria (o seu custo de produção em termos de salários), era inferior ao "trabalho comandado" (aquilo que a mercadoria podia, uma vez vendida, "comprar" em termos de horas de trabalho). Para Smith, esta discrepância é que explicava a existência do lucro, mas não suas causas. Smith considerava que o lucro estava associado à propriedade privada do capital, na medida em que a renda de um empresário dependia menos do seu trabalho como gerente do que do volume dos seus investimentos, mas tal não explicava a existência do lucro como um overhead sobre os custos de produção em termos de salários. Uma das saídas que Smith considera, é que lucro é proveniente da oferta e da procura. Ou seja, o lucro é criado pelo mercado. Distancia o lucro (riqueza) do processo de trabalho.

Para Marx (ibid.) a o lucro saía diretamente do produto do trabalhador, no que chamou de "mais-valia", ou seja, a apropriação de um excedente de trabalho do trabalhador pelo patrão (SEWELL, 2013).

De qualquer modo, pode-se entender que o aumento da receita relaciona-se ao output da firma e que o aumento no lucro se encontra de algum modo relacionado ao aumento da diferença entre a receita e do que se gasta para obtê-la. Por sua vez, a produção relacionase com a receita, e como afirmava Kantorovich (1960), os meios de aumentar a produção residem no aumento da tecnologia ou na organização da produção.

Portanto, este artigo explica o lucro também como resultado do incremento da tecnologia associado à utilização de máquinas, a partir de argumentos matemáticos e de um modelo interativo criado com base nos modelos clássicos de programação matemática.

\section{MODELO MATEMÁTICO}

Adota-se, para o presente estudo, o modelo do valor trabalho com fronteiras de possibilidades de produção (FPP), de modo a ser fiel ao pensamento dos clássicos que se quer analisar (Ricardo, Adam Smith e Marx).

Uma FPP designa o lugar geométrico das quantidades máximas de cada bem passíveis de serem produzidas por cada produtor, segundo sua dotação de fatores, conforme Carvalho e Silva (2007, p. 33).

$$
L=a_{1} x_{1}+a_{2} x_{2}+\ldots+a_{n} x_{n}
$$

Como o único insumo é o trabalho, chama-se de $L$ o montante de todo o trabalho do produtor $i$, considerando-se máquinas associadas à tecnologia. Chama-se de $L_{D}$, todo os homens horas de todos os trabalhadores do produtor.

\section{DIGRESSÃO SOBRE AS VARIÁVEIS ENVOLVIDAS NO PROCESSO}

Neste item do artigo, apresentam-se as variáveis envolvidas no desenvolvimento a seguir de modo a prover uma melhor conceituação e indicar as unidades em que se computam seus valores.

Preliminarmente, esclarece-se que são taxas temporais, entretanto, não se indica a dependência no tempo, porque as relações que exprimem são em um dado momento, como se congeladas em uma fotografia. Outrossim, pode-se dizer que existe uma correspondência entre o número de máquinas e o número de produtos (um isomorfismo), isto porque cada máquina produz um único produto.

Assim, discorre-se sobre cada uma:

(1) $a_{i}$ - o coeficientes técnicos de produção, no caso desta demonstração particular, relaciona um único produto que é o valor trabalho (que pode facilmente ser convertido 
em dinheiro mediante a multiplicação do valor do homem hora em reais pelo montante de homens-hora. Este coeficiente tem a unidade $\mathrm{HH}$ /quantidade. A unidade de quantidade é referente à unidade de medida apropriada, $\mathrm{kg}$ - se massa, litros - se volume, etc. O coeficiente técnico de produção, neste caso, expressa matematicamente na contabilidade de uma máquina que converte o insumo em produto (com o valor de $\mathrm{HH}$ ), incorporando a tecnologia ao trabalho, com a meta de se obter efeito multiplicador;

(2) $x_{i}$ - quantidade produzida da mercadoria $i$, novamente, A unidade de quantidade é referente à unidade de medida apropriada, $\mathrm{kg}$ - se massa, litros - se volume, etc;

(3) $L$ - reserva de trabalho é um valor dado em HH. A curva que apresenta a FPP é uma projeção no hiperplano das quantidades da hipersuperfície $L\left(x_{1}, x_{2}, \ldots, x_{n}\right)$;

(4) $L_{D}$ - trabalho disponível (oferecido ou fornecido pelos trabalhadores) também é dado em $\mathrm{HH}$, entretanto, representa o número de $\mathrm{HH}$ que todos os empregados (termo genérico) podem disponibilizar, sem perdas (como afastamentos, etc). Este valor é tautologicamente igual ao número de empregados multiplicado pelos $\mathrm{HH}$ que podem fornecer;

(5) $R$ - representa o número de horas que cada trabalhador pode trabalhar. Como dito anteriormente, este número é uma taxa temporal, a rigor seria o número de $\mathrm{HH} / \mathrm{dia}$. Como todos os cálculos são por dia, simplifica-se sua medida para a unidade $\mathrm{HH}$;

(6) $t_{i}$ - tecnologia empregada na produção do produto i. Como, por simplificação, toda a tecnologia está inserida na máquina, a máquina se apresenta como continente de uma determinada tecnologia que, se for maior representa maior volume de produção. Infelizmente, não corresponde sempre à verdade, às vezes, a tecnologia vem para melhorar a qualidade e não a quantidade, o que se considerou não prejudica o modelo;

(7) $\lambda_{i}$ - número de trabalhadores necessário para se fazer um produto. Este número é igual em qualquer firma e em qualquer lugar, pois se consideram os trabalhadores os mesmos em qualquer lugar. Este modelo diferencia os $\mathrm{HH}$ empregados por uma firma através da razão $\lambda_{i} / t_{i}$, que é diferente em cada lugar. Além disso, o modelo não considera as diferenças entre outros fatores de produção.

A diferença $L-L_{D}$ representa a diferença entre o trabalho fornecido e o trabalho necessário para criação de todos as quantidades de produtos que sejam produzidas por um produtor.

\section{HIPÓTESES}

No desenvolvimento que se procede a seguir, consideram-se as seguintes hipóteses:

(h.1) $a_{i}<1$, de modo a fazer com que haja um efeito multiplicador da quantidade produzida;

(h.2) $t_{i}>1$, pelas mesmas razões de (h.1);

(h.3) A tecnologia utilizada do produtor na produção do produto $i$ é definida por $t_{i}$ e é o inverso do coeficiente de insumo produto, expresso matematicamente, por (2).

$$
a_{i}=\frac{R \lambda_{i}}{t_{i}}
$$

\section{TECNOLOGIA E COEFICIENTES TÉCNICOS}

Define-se o excedente de produção como lucro, ou seja, o excedente entre a capacidade disponível de mão-de-obra e a o homem-hora gerado com o uso de máquinas, ou seja, a reserva de trabalho da fronteira de produção. A figura 1 apresenta uma ilustração do coeficiente $a_{i}$, entendido como uma função. 


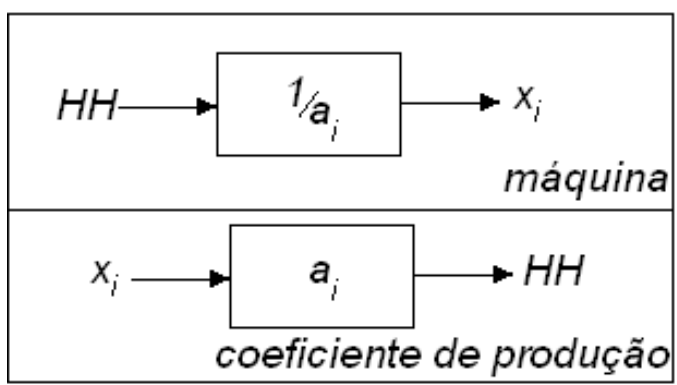

Figura 1- Dualidade máquina - coeficiente técnico de produção

Com o uso desta definição no modelo (3), o lucro pode ser calculado percentualmente como a razão (adimensional) entre o trabalho disponível em $\mathrm{HH}$ e a reserva de trabalho. Assim, pode-se chegar a (4):

$$
\begin{gathered}
L_{D}=R\left(\lambda_{1}+\lambda_{2}+\ldots+\lambda_{n}\right) \\
\frac{L-L_{D}}{L}=\frac{R}{L}\left[\lambda_{1}\left(\frac{x_{1}}{t_{1}}-1\right)+\lambda_{2}\left(\frac{x_{2}}{t_{2}}-1\right)+\ldots+\lambda_{n}\left(\frac{x_{n}}{t_{n}}-1\right)\right]
\end{gathered}
$$

Para identificar o sinal e a magnitude da expressão (4), passa-se a analisar os termos que aparecem em parênteses, fazendo que $x_{1}=x_{2}=\ldots=x_{n}=1$.

$$
\left(\frac{L-L_{D}}{L}\right)_{x_{1}=\ldots=x_{n}=1}=\frac{R}{L}\left[\lambda_{1}\left(\frac{1}{t_{1}}-1\right)+\lambda_{2}\left(\frac{1}{t_{2}}-1\right)+\ldots+\lambda_{n}\left(\frac{1}{t_{n}}-1\right)\right]
$$

Como, naturalmente, cada coeficiente $a_{i}$ é menor que 1 , necessariamente, pois incorpora a tecnologia ao trabalho de modo a se ter um efeito multiplicador, então pode-se afirmar que

$$
\frac{L-L_{D}}{L}<0 \Leftrightarrow 1-\frac{1}{t_{i}} \geq 0, i=1 \ldots n
$$

Com isso, percebe-se que existe um excesso de trabalho que tanto (como mais comumente) é fruto do emprego de máquinas e tecnologia. Este excesso de trabalho é um excesso de receita $\left(L-L_{D}\right)$ em dinheiro em relação à despesa com os salários dos empregados $L_{D}$

Esta conclusão confere com a experiência histórica que mostra que conforme a tecnologia têm se enraizado no seio produtivo, mais os trabalhadores (que só possuem trabalho disponível) se têm distanciado dos capitalistas (que possuem máquinas).

\section{MAXIMIZAÇÃO DO LUCRO}

Naturalmente, surge a pergunta de qual deve ser a estratégia da empresa para maximizar o lucro, se aumentar a tecnologia ou reduzir os salários.

Fazendo-se uso de um programa simplificado de programação matemática podese atingir facilmente este resultado.

$$
\begin{aligned}
& \min L-L_{D} \\
& \lambda_{1}, \ldots, \lambda_{n} ; t_{1}, \ldots, t_{n}, L_{D} \geq 0
\end{aligned}
$$

Que pode ser convertido no programa (8), com a aplicação do método dos multiplicadores de Lagrange: 


$$
\Lambda=R x_{1}\left(\frac{\lambda_{1}}{t_{1}}\right)+R x_{2}\left(\frac{\lambda_{2}}{t_{2}}\right)+\ldots+R x_{n}\left(\frac{\lambda_{n}}{t_{n}}\right)-\theta_{0} R\left(\lambda_{1}+\lambda_{2}+\ldots+\lambda_{n}-\frac{L_{D}}{R}\right)-\sum_{i=1}^{n}\left[\theta_{i}\left(\lambda_{i}+s_{i}\right)+\pi_{i}\left(t_{i}+\sigma_{i}\right)\right]-\mu\left(\frac{L_{D}}{R}+\varepsilon\right)
$$

O que leva ao conjunto de equações (9a) a (9i):

$$
\begin{aligned}
& \frac{\partial \Lambda}{\partial \lambda_{i}}=\left(\frac{R x_{i}}{t_{i}}\right)-\theta_{0} R-\theta_{i}=0, i=1 \ldots n \\
& \frac{\partial \Lambda}{\partial t_{i}}=-\left(\frac{R x_{1}}{t_{i}^{2}}\right)-\pi_{i}=0, i=1 \ldots n \\
& \frac{\partial \Lambda}{\partial s_{i}}=-\theta_{i}=0, i=1 \ldots n \\
& \frac{\partial \Lambda}{\partial \sigma_{i}}=-\pi_{i}=0, i=1 \ldots n \\
& \frac{\partial \Lambda}{\partial \theta_{0}}=R\left(\lambda_{1}+\lambda_{2}+\ldots+\lambda_{n}-\frac{L_{D}}{R}\right)=0, i=1 \ldots n \\
& \frac{\partial \Lambda}{\partial \theta_{i}}=\left(\lambda_{i}+s_{i}\right)=0, i=1 \ldots n \\
& \frac{\partial \Lambda}{\partial \pi_{i}}=\left(t_{i}+\sigma_{i}\right)=0, i=1 \ldots n \\
& \frac{\partial \Lambda}{\partial L_{D}}=-\theta_{0} R-\frac{\mu}{R}=0, i=1 \ldots n \\
& \frac{\partial \Lambda}{\partial \mu}=\frac{L_{D}}{R}+\varepsilon=0, i=1 \ldots n
\end{aligned}
$$

Resolvendo o sistema (9a) a (9i), tem-se:

$$
\begin{aligned}
& \theta_{i}=\pi_{i}=\mu=0, i=1 \ldots n \\
& \lambda_{i}=-s_{i}, i=1 \ldots n \\
& t_{i}=-\sigma_{i}, i=1 \ldots n \\
& L_{D}=-R \varepsilon \\
& \left(\frac{R x_{1}}{t_{i}^{2}}\right)=0 \Rightarrow t_{i} \rightarrow \infty
\end{aligned}
$$

Conclui-se, como já se sabia desde o princípio, que o problema é ilimitado em tecnologia, ou seja, a solução ótima do lucro (o lucro máximo) encontra-se quando, independentemente do número de empregados, a tecnologia é a máxima possível.

\section{OBSERVAÇÕES ACERCA DO ARTIGO APÓS SUA REVISÃo}

Após os comentários dos revisores, o artigo melhor estabeleceu o conceito de mais-valia e o diferenciou do lucro, no sentido de que a mais-valia passou a ser, como tradicionalmente definida (SEWELL, 2013), apenas o excesso de trabalho do empregado apropriado pelo patrão, e o lucro a diferença entre o trabalho disponível e o trabalho gerado com o uso das máquinas. Assim, muitas frases em que a palavra mais-valia aparecia com o sinônimo de lucro foram alteradas para lucro e vice-versa.

Uma importante consequência desta distinção é que ela destacou o fato de que todo o texto está sob o ponto de vista de uma empresa e seus trabalhadores, que veem a 
sociedade como ambiente externo, ou seja, fora de seu sistema. Além disso, o presente artigo não discute o mérito da teoria marxista da mais-valia, nem da queda tendencial da taxa de lucro.

$\mathrm{Na}$ verdade, indiretamente, o modelo mostra a validade da queda tendencial da taxa de lucro, considerando-se que, explicitamente, na equação (4), quanto menor o número de trabalhadores empregado na produção da mesma quantidade, tanto menor o lucro. Historicamente, por outro lado, o capitalismo tem feito reduções constantes de mão-de-obra na produção de quantidades de produtos, o que vem dizer com outras palavras que existe uma queda tangencial na taxa de lucro.

Outra importante consequência dos comentários dos revisores, embora não tenha sido sua orientação, foi a inclusão no título da palavra tecnologia, que para o autor parecia ser necessário a melhor representar o conjunto de ideias apresentadas.

Enfim, pelas críticas recebidas, este autor gostaria de agradecer aos revisores.

\section{CONCLUSÃO}

Apresenta-se uma discussão alternativa para as origens do lucro, a partir de um modelo simplificado de programação matemática.

Para tanto, mostra-se que existe um excesso de trabalho que tanto (como mais comumente) pode ser fruto da tecnologia, como do emprego do excesso de trabalho do trabalhador (ou falta de remuneração adequada, a mais-valia).

Esta conclusão confere com a experiência histórica que mostra que, conforme a tecnologia têm se enraizado no seio produtivo, como atesta a revolução industrial, mais os trabalhadores (que só possuem trabalho disponível) têm-se distanciado dos capitalistas, que possuem máquinas.

Neste modelo, percebe-se que, independentemente da quantidade de mão-de-obra, o máximo de lucro se encontra no emprego máximo de tecnologia.

Enfim, todo o texto está sob o ponto de vista de uma empresa e seus trabalhadores, que veem a sociedade como ambiente externo, ou seja, fora de seu sistema. Além disso, o presente artigo não discute o mérito da teoria marxista da mais-valia, nem da queda tendencial da taxa de lucro.

\section{REFERÊNCIAS BIBLIOGRÁFICAS}

CARVALHO, M. A.; SILVA, C. R. L. Economia Internacional. 4ª Ed., São Paulo: Saraiva, 2007.

KANTOROVICH, L. V. "Mathematical Methods of Organizing and Planning Production" Management Science, Vol. 6, No. 4 (Jul., 1960), pp. 366-422. Disponível em: http://www.jstor.org/stable/2627082 Acesso: 8 jan. 2014.

MAIS-Valia. In: WIKIPEDIA: a enciclopédia livre. [1999?]. Disponível em: < http://pt.wikipedia.org/wiki/Mais-valia>. Acesso em: 28 nov. 2013.

SEWELL, Rob. "A crise capitalista e a queda tendencial da taxa de lucro - Primeira parte". [s.l]:[[s.n], 2013 (data provável). Disponível em: http://www.marxismo.org.br/content/crisecapitalista-e-queda-tendencial-da-taxa-de-lucro-primeira-parte Acesso: 15 jun. 2014.

WILliAMSON, J. A Economia Aberta e a Economia Mundial: Um Texto de Economia Internacional. Tradução de José Ricardo Brandão Azevedo, São Paulo: Campus, 1988. 